\title{
Current situation and development trend of traceability system of hazardous chemicals in China
}

\author{
Teng Su${ }^{1}$, Chang Liu ${ }^{1}$ and Ru Zhou ${ }^{1 *}$ \\ ${ }^{1}$ College of Safety Science and Engineering, Nanjing Tech University, Nanjing, Jiangsu, 211816, China
}

\begin{abstract}
The traceability system of hazardous chemicals is a kind of information identification and tracking technology, which mainly involves the collection and management of the whole life cycle and whole chain information of hazardous chemicals. As an important means of safety supervision of hazardous chemicals, it is conducive to the long-term development of enterprises and the improvement of public safety. At present, the safety situation of China's chemical industry is grim. It is urgent to establish a practical and effective traceability system of hazardous chemicals to eliminate the obstacles of information acquisition of hazardous chemicals, strengthen safety supervision and enhance the ability of emergency response. This paper summarizes the development process, Traceability Technology and traceability development trend of China's hazardous chemicals traceability system, and puts forward some suggestions for improving and perfecting China's hazardous chemicals traceability system.
\end{abstract}

\section{Introduction}

As an important basic industry of national economy, the development of chemical industry is closely related to the form of national economy Since the 12th Five Year Plan, China's chemical industry has maintained rapid development, and the industry's income and profit scale have grown rapidly. Data show that from 2008 to 2019, China's GDP will grow from 26447.3 billion yuan to 99066.5 billion yuan, with an average annual compound growth rate of $12.77 \%$. By the end of June 2020, there are 22745 Enterprises above Designated Size in the chemical industry, involving nearly 4000 kinds of hazardous chemicals, most of which are flammable, explosive, toxic and harmful ${ }^{[1]}$. With the rapid development of the national economy, the output and consumption of hazardous chemicals are also increasing. The risk of chemicals in the production, storage and transportation, use and disposal of waste and other stages of the whole life cycle is also increasing. The role of the chemical industry in promoting social development is becoming more and more important. At the same time, its possible harm can not be ignored. For hazardous chemicals, once improper supervision occurs, the harm will be extremely huge, which will not only cause a lot of economic losses, but also endanger personal safety.

The concept of traceability was first put forward and applied in automobile, aerospace and other fields ${ }^{[2]}$. Since then, around the traceability, a series of operation specifications and standard systems have been popularized in the industry. With the rapid development of traceability related research and application, all kinds of related information in the whole life cycle of hazardous chemicals can be taken as the research object. The frequent occurrence of hazardous chemical safety accidents has sounded an alarm for all stakeholders of hazardous chemical safety, such as government departments and production enterprises, which makes people urgent to strengthen the control of all-round source information of hazardous chemical products and better realize the effective traceability of hazardous chemical products. The frequent safety incidents of hazardous chemicals threaten the public safety of the people, the effective supervision of government departments and the operation and development of enterprises all the time. More and more countries and regions put forward rigid requirements for the establishment of traceability system of hazardous chemicals. Once it has the ability of traceability, in case of safety problems of hazardous chemicals, government departments can find the problems at the first time and implement timely and effective rescue supervision; Enterprises can also quickly take measures to minimize losses; In addition, having the ability to trace the source of hazardous chemicals will greatly improve the safety level of hazardous chemicals. In conclusion, traceability of hazardous chemicals is gradually becoming a research hotspot to ensure the safety of hazardous chemicals and improve the level of chemical safety.

\section{Development status of hazardous chemicals traceability system}

\subsection{Development background of hazardous chemicals traceability system}

The content of traceability mainly covers two aspects: one

*Corresponding author's e-mail: maxmuse.zhou@njtech.edu.cn 
is backtracking, which mainly explores the process of products along the production chain and transportation chain, with the purpose of constructing the circulation history of products; The second is tracking, which is mainly to track products along the production chain and transportation chain, and its purpose is to carry out realtime monitoring of product risk. Traceability was born in a series of food safety crises (mad cow disease in England, Salmonella contamination in pork in Denmark, etc.). Some food safety problems such as these strongly promote the development of traceability system. In 2002, some EU countries proposed to establish a food traceability system, which is a food safety information regulatory system for the purpose of controlling food hazards, ensuring fair trade and public health. Since then, the traceability system of all walks of life has emerged.

In early 2016, the "opinions of the general office of the State Council on accelerating the construction of traceability system for important products" made a scientific plan for the traceability system of hazardous chemicals, and proposed to focus on the supervision of civil explosives, explosive prone dangerous chemicals and highly toxic chemicals, so as to carry out the whole life cycle traceability system construction for these hazardous substances ${ }^{[3]}$. In the world, it is very important to establish an effective traceability system for hazardous chemicals. In view of the dangerous characteristics of hazardous chemicals, such substances are prone to cause safety accidents due to poor supervision, and the accidents are serious. For those hazardous chemicals with large circulation, special control is needed. Taking China as an example, the traceability system of hazardous chemicals in China is not perfect, and with the occurrence of safety incidents related to hazardous chemicals in recent years, the public's trust in hazardous chemicals is gradually losing. It is urgent to take standardized measures to ensure the safety of hazardous chemicals market, which requires us to adopt advanced management mode and scientific technical means.

\subsection{Research Progress on Traceability Technology of hazardous chemicals}

The main traceability technologies are mainly two types, namely, the information recording technology for coding data and the information authentication technology for capturing the material ontology attributes. The former mainly refers to the subjective extraction of the specified information from manufacturing to abandonment of the whole life cycle of the product through proprietary coding, and then carrying and recording through relevant technologies, and finally realizing the tracing and query of the traceability information. The advantages of this method are low threshold of practice and easy to spread. Short board is that information can be modified manually. When the media of information recording is separated from the ontology, tracing will be difficult. The latter is mainly through the acquisition of the product ontology attribute characteristics and the production stage of the natural information to be associated, and then realize the origin of the product traceability. The main advantage is that the origin traceability can be realized by establishing the feature database based on the attribute data and the information of the origin under the unknown scenario. High cost, high threshold and the traceability precision to be improved are the main obstacles to popularization.

\subsubsection{Information coding technology}

From the perspective of easy practice, such as the threshold and reproducibility of technology, most of today's traceability systems are based on coding technology. Information coding is the process of using a symbol language system which is convenient for human and machine recognition to express information. At this stage, many European and American countries have adopted EAN-UCC system to complete the establishment and application of traceability system for many products, but there is an unavoidable short board in the system, that is, when the transmission chain of a certain link breaks, the transmission of information will stop immediately ${ }^{[4]}$. In addition to this technology, there are other information coding recording technologies, such as electronic data interchange (EDI) and bar code technology. EDI technology is mainly realized by electronic means, which converts data information into standard information, and then carries out automatic interaction between computers. The realization process of EDI technology is completely automatic or can be realized with the least manual intervention ${ }^{[5]}$.

The principle of RFID technology is: the tag is placed on the traced product, and the tag can be activated by the reader that emits the radio wave of a specific frequency band. After being activated, the tag will immediately return the data to the reader. At the same time, the information processing control system will also make a decision in the first implementation. The technology has been formally applied in the field of domestic food products, such as meat production and organic product certification [6]. However, RFID is still a kind of information recording technology in essence, and the input process of traceability data still cannot get rid of the dependence on people. In addition, the product body and label are in a state of physical separation, which leaves hidden danger for human subjective operation and the objective environment of hazardous chemical chain, and it is difficult to eliminate the hidden danger. At the same time, RFID products in the promotion and application of origin traceability also face some other problems, such as high cost and technical standards are not unified ${ }^{[7]}$.

\subsubsection{Analysis technology of hazardous chemicals}

The traceability system based on information coding recording technology aims to ensure the continuity of records, but now more and more attention is focused on exploring a technical way based on the natural properties of the material itself to explore the origin of the material and judge the authenticity of the material, and strive to minimize the impact of interference factors through scientific and technical means, At the same time, when other technologies can not play a role, it becomes an 
important tool to support the safety of hazardous chemicals and realize scientific and effective traceability. This kind of technology mainly obtains the attribute data of hazardous chemicals ontology through a variety of analytical chemistry methods. These data are deeply associated with the natural information of hazardous chemicals producing areas (production process of hazardous chemicals, geographical characteristics of various raw materials, etc.), and then assisted by chemometrics, so as to realize the origin traceability of hazardous chemicals.

The focus of the research on the Origin Traceability Technology of hazardous chemicals is to explore the information indicators that contribute to the origin differences. If suppliers use different initial crude oil mixtures, it can be expected that there will be significant differences in isotope ratios of crude oil sold by different suppliers ${ }^{[8]}$. These analytical techniques mainly include a variety of analytical techniques such as chromatography and mass spectrometry ${ }^{[9]}$. In terms of the application of chromatographic technology, high performance liquid chromatography (HPLC) can be used to effectively analyze a variety of components in products, and can also provide more investigation indicators for the origin traceability of dangerous chemicals. Its application in the origin identification of wine and dairy products has been reported ${ }^{[10,11]}$. In the application of mass spectrometry technology, stable isotope ratio mass spectrometry technology is widely used in the origin tracing and identification of products. At first, it was used in the research of food identification, and then it was successfully used in the traceability of crude oil, diesel oil and other compounds ${ }^{[12-14]}$. This technology can trace the origin of materials by identifying the unique isotopic geographical characteristics of the origin of materials, which is similar to the unique "fingerprint" of human body, and has great guiding significance for the origin traceability of various products (including hazardous chemicals). The recent advances in terms of hazardous chemicals traceability technology are summarized in Table 1.

Table 1. Technological advancement.

\begin{tabular}{l|l|l}
\hline Technology & Concept & Author \\
\hline & The source of crude oil can be traced accurately & Liu X.X. (2012) \\
Isotope analysis & The potential of this technology to distinguish fuel is proved & Harvey S D. (2012) \\
& The discrimination efficiency of diesel samples is improved & Novák M.(2017) \\
\hline
\end{tabular}

\subsection{Application of hazardous chemicals traceability system}

At present, at home and abroad, tracing the source of hazardous chemicals mainly focuses on those hazardous chemicals with large circulation and need special control, such as fuel oil and common solvents. With the release of "opinions of the general office of the State Council on accelerating the construction of traceability system for important products" in 2016, the construction and application of traceability system for chemicals has been launched in China. In May 2017, Beijing Bureau of quality supervision arranged and deployed the pilot work plan for the construction of traceability system for dangerous goods and other important chemical products, and discussed the possibility of carrying out informatization traceability pilot demonstration with Beijing chemical reagent and vehicle urea aqueous solution production enterprises as the main body. In 2019, the State Administration of market supervision and administration and the State Standardization Administration issued the basic requirements for the traceability system of important products (GB / T 38158-2019), which specifies the general requirements for the product traceability system (system construction, data management, system operation and maintenance, etc.). In the first half of 2020, the Ministry of emergency management, the Ministry of industry and information technology, the Ministry of public security and the Ministry of transport jointly released the catalogue of specially controlled hazardous chemicals (First Edition), which gave instructions on the construction of information platform and the implementation of full life cycle traceability control of hazardous chemicals ${ }^{[15]}$. However, in general, China has not yet established a comprehensive and effective traceability system for hazardous chemicals. At present, only a small number of hazardous chemicals producing areas and large hazardous chemicals manufacturers have made attempts to establish a smallscale traceability system. For example, in 2019, Shijiazhuang City, Hebei Province, took the lead in establishing a "hazardous chemicals traceability system" in the country. The system makes full use of the information platform to achieve source traceability the destination can be inquired and supervised in an all-round way. It can be seen that the traceability system of hazardous chemicals has become an effective means to protect people's lives and health and maintain public safety. At present, all countries in the world have realized its irreplaceable importance, and are competing to invest a lot of human and material resources to continuously promote the improvement of the system. 


\section{Research significance and development trend of hazardous chemicals traceability system}

China is the major producer and consumer of hazardous chemicals in the world. By the end of June 2020, 22745 enterprises in the chemical industry have reached a scale, with nearly 4000 kinds of dangerous chemicals involved. Most of these dangerous chemicals are inflammable, explosive, toxic and harmful ${ }^{[16]}$. Each brand of hazardous chemicals is produced under specific process conditions with specific raw materials in a specific area. Some products with high-end raw materials and processes have higher quality and higher market price than general products. And because of the domestic level of hazardous chemicals safety management and the start of risk prevention and control technology is relatively late. Driven by economic benefits, some illegal businesses use false labels to fake high-quality products, store them illegally and transport them in excess of quantity, which cause great damage to the brand of high-quality products; Serious accidents of hazardous chemicals may even be caused, which may endanger public safety. In China, government agencies pay more and more attention to the control of dangerous chemicals. The overall reason is that although the potential of chemical industry market in China is huge, there are many hazardous chemical products, such as the "8.12" fire and explosion accident in Tianjin port and the special major explosion accident of "3.21" of the sound water, which have exposed many deficiencies in the control of dangerous chemicals compared with those of developed countries in Europe and America ${ }^{[17]}$. When similar hazard accidents occur, after the rescue, it is necessary to analyze the causes of the accident and trace back the specific responsible persons.

Although the potential of chemical industry market in China is huge, there are still many shortcomings in the safety of dangerous chemicals. The domestic safety management level and risk prevention and control technology started late. There are always some illegal businesses to increase revenue and reduce costs, illegal storage, excessive transportation and reduce safety investment. These factors may lead to the occurrence of hazardous chemicals accidents. The common dangerous chemicals safety incidents have brought great threat to the development of hazardous chemicals enterprises, the public life safety and the effective supervision of the government. The establishment and improvement of traceability system of hazardous chemicals is becoming the more and more hard regulation of the country. After having traceability ability, in case of safety problems of hazardous chemicals, the enterprise will be able to take measures to reduce losses in the first time; The government can also find problems in time and implement effective supervision; In addition, it can trace the source of dangerous chemicals, which will guarantee the safety level of dangerous chemicals to a great extent.

In view of the problem of tracing the origin of dangerous chemicals, many countries have made many laws and regulations. In the comprehensive treatment plan for hazardous chemicals safety and the catalogue of special control of hazardous chemicals, China also clearly places the control and control of some bulk chemical raw materials in a very important position. These regulations clearly define the control measures needed to carry out the traceability of hazardous chemicals, such as building information platform for hazardous chemicals, implementing information tracing of the whole life cycle of hazardous chemicals, packaging dangerous chemicals uniformly, fully implementing the access standards for safe production, strengthening the transportation supervision of hazardous chemicals, etc., so as to minimize the safety risk and effectively prevent and curb serious accidents, To ensure the safety of people's lives and property effectively ${ }^{[18]}$.

\section{Conclusions}

In conclusion, from the perspective of industrial and social needs, it is of great significance to establish and improve the traceability system of hazardous chemicals, which is of great significance to improve the supervision ability of government departments on the production and storage and transportation of hazardous chemicals and to ensure public safety; As far as the enterprise is concerned, it can make its own brand sound and realize the high added value of the product. At present, the main tracing technology in the world is to implement traceability through information recording and coding system. After analyzing the current situation of our country, we can find that the traceability system of hazardous chemicals in China is still in the development stage, and the Traceability Technology and system regulations still need to be improved, so it is a long way to establish and perfect the traceability system of dangerous chemicals.

\section{Acknowledgments}

This research was supported by National Key Research and Development Program of China (No.2018YFC0809300).

\section{References}

1. Cao X.P. Development status, problems and Countermeasures of chemical industry park $[\mathrm{J}]$. Chemical management, 2020(07):1-3.

2. Montalván R, Ando A, Echeverrigaray S. Use of seed protein polymorphism for discrimination of improvement level and geographic origin of upland rice cultivars[J]. Genetics and Molecular Biology, 1998, 21 (4) :531-535.

3. Anonymity. Information traceability system of dangerous goods and other important products to be built in China [J]. Low temperature and special gas, 2017(01):30.

4. Kong H.L, Li J.H. Application of global unified identification system in food safety tracking and traceability system [J]. Food science, 2004(06):188194. 
5. Liu W.G, Bai Y.Z. Development and application of bar code technology [J]. Digital world , 2018,000(005):504-505.

6. Guo W, Chu X.G. RFID technology and its application in food safety field [J]. Food technology, 2007(09):5-7.

7. Qu A.L, Liu H.M, Ma C.L. Application of RFID technology in food traceability [J]. Agricultural products processing, 2020(08):86-88.

8. Smallwood B J, Philp R P, Allen J D. Stable carbon isotopic composition of gasolines determined by isotope ratio monitoring gas chromatography mass spectrometry[J]. Organic Geochemistry, 2002,33(2): 149-159.

9. Wei Y.M, Guo B.L, Wei S, et al. Research and application methods of food origin traceability and confirmation technology [J]. Chinese Agricultural Sciences, 2012, 45(24):5073-5081.

10. Laureles L R, Rodriguez F M, Reaño C E, et al. Variability in fatty acid and triacylglycerol composition of the oil of coconut (Cocos nucifera L.) hybrids and their parentals. [J]. J Agric Food Chem, 2002, 50(6):1581-1586.

11. Cunha, S. C, Faria, et al. Quantification of organic acids in grape musts and Port wines[J]. Ciencia y Tecnología Alimentaria, 2002, 3(4) :212.

12. Liu X.X, Wang Y, Wang Y.L, et al. Identification of Middle East crude oil by carbon stable isotope ratio mass spectrometry [J]. Analytical chemistry, 2012, 40 (07) :1104-1108.

13. Harvey S D, Jarman $\mathrm{K} \mathrm{H}$, Moran J J, et al. Characterization of diesel fuel by chemical separation combined with capillary gas chromatography (GC) isotope ratio mass spectrometry (IRMS)[J]. Talanta, 2012, 99:262-269.

14. Novák M, Palya D, Bodai Z, et al. Combined cluster and discriminant analysis: An efficient chemometric approach in diesel fuel characterization[J]. Forensic Science International, 2017, (270):61-69.

15. Journal editorial department. Four departments jointly implement special control on 20 kinds of dangerous chemicals [J]. Safety production in China, 2020, 15(07):6-7.

16. Zhou F.J. Research on safety management of hazardous chemicals in chemical industry[D]. 2012, Yunnan University, MA thesis. pp. 4-7.

17. Zong H. How to manage dangerous chemicals abroad [J]. Fire protection in China, 2015(19):48-49.

18. Ying J.W. Strict examination and approval of projects, strict personnel access, storage and fixed location management, special state control of 20 categories of dangerous chemicals [J]. Shanghai Chemical Industry, 2019, 44(11):41. 